\title{
Caracterização e avaliação de reservas geológicas do depósito mineral de Farim-Saliquinhé
}

\section{Characterization and evaluation of the geological reserves in the Farim-Saliquinhé mineral deposit}

\section{Gilberto Charifo}

Engenheiro de Minas e Mestre em Engenharia Geológica, Sócio Gerente da Gold-Fluvium, Rio Maior, Portugal

E-mail:geral@gold-fluvium.com

\section{José A. Almeida}

Doutorado em Engenharia de Minas, Professor do DCT e Investigador do CICEGe, FCT-Universidade Nova de Lisboa, Monte da Caparica, Portugal E-mail: ja@fct.unl.pt

\section{Resumo}

Esse trabalho apresenta uma metodologia integrada e os principais resultados da modelagem geológica e econômica do jazigo mineral de fosfato de FarimSaliquinhé, Guiné-Bissau. Esse jazigo foi, primeiramente, reconhecido nos anos 80, pelo Bureau de Recherches Géologiques et Minières (BRGM), como sendo de origem sedimentar.

A caracterização morfológica consistiu na estimação da profundidade e da espessura da camada de estéril e das formações mineralizadas (rochas fosfáticas FPA, principais, e carbonato-fosfática FPB, secundária). A caracterização dos teores em $\mathrm{P}_{2} \mathrm{O}_{5}$ foi efetuada para uma malha de blocos por krigagem e, posteriormente, por simulação, para caracterizar a incerteza e classificar as reservas em inferidas, indicadas ou medidas.

A morfologia e os teores foram, posteriormente, convertidos em benefícios/ custos, conforme um potencial cenário de exploração mineral. No que se refere à modelagem econômica, apresenta-se um estudo de pré-viabilidade econômica, com análise de sensibilidade, relativamente aos parâmetros preço de venda, custo de extração, custo de transporte e desconto no preço de venda da rocha fosfática por não concentração e uma estimativa da taxa interna de retorno (TIR) do projecto para um cenário-base e uma variante.

Palavras-chave: Fosfato, Farim-Saliquinhé, avaliação de reservas, krigagem, teor de corte, curvas de parametrização, estudo de pré-viabilidade econômica.

\footnotetext{
Abstract

This article presents an integrated methodology and main results of the geological and economic modeling of the Farim-Saliquinhé phosphate ore deposit, in Guiné-Bissau. This mineral mass was first recognized in the 1980s, by the Bureau de Recherches Géologiques et Minières (BRGM) as sedimentary.

The morphological characterization was performed by depth and thickness estimation of coverage and mineralized formations (main phosphate rock FPA, and carbo-phosphate FPB, secondary). The $\mathrm{P}_{2} \mathrm{O}_{5}$ grades were characterized by krigging and subsequently by geostatistical simulation, to infer the uncertainty and classify the reserves in terms of inferred, indicated and measured.
} 
Finally, the morphology and grades were converted into benefits and costs following a mineral exploitation scenario. With regard to the economic modeling, a pre-feasibility study is presented with sensitivity analysis, relative to the phosphate price, exploitation and transportation costs, and reduction on the phosphate price, if not concentrated. An estimate is also presented of the internal rate of return (IRR) of the project for a base scenario and one variant.

Keywords: Phosphate, FarimSaliquinhé, ore reserves evaluation, krigging, cut-off grade, cumulative and grade curves, pre-feasibility study.

\section{Introdução}

Os fosfatos formam um grupo de minerais não metálicos, cujo componente principal é o elemento fósforo. Encontram-se em depósitos minerais de origem sedimentar, ígnea ou biogenética (Notholt, 2005; Velho, 2005). Os depósitos sedimentares e os depósitos de origem ígnea são os mais importantes do ponto de vista econômico. Os depósitos biogenéticos são concentrações orgânicas nitrogenadas, originadas pelos dejetos de aves, e têm menor importância econômica.

Na Guiné-Bissau, nos anos 80, foi reconhecido pelo Bureau de Recherches Géologiques et Minières (BRGM) um depósito mineral de fosfato, posteriormente denominado Farim-Saliquinhé, de origem sedimentar (BRGM, 1983). Esse depósito localiza-se a menos de 3 km da povoação de Farim, junto ao rio Cachéu (Figura 1). No estudo do BRGM foram efetuadas mais de 80 sondagens na região e um levantamento topográfico da superfície.

As reservas totais de rocha fosfática foram estimadas em 120 milhões de toneladas, com teor médio em $\mathrm{P}_{2} \mathrm{O}_{5}$ de $30 \%$, de acordo com os dados e a metodologia de avaliação utilizada em 1986. Esse depósito encontra-se atualmente concessionado para uma possível exploração mineira pelo que se justifica o presente trabalho de modelação, avaliação de reservas e estudo de préviabilidade econômica.

O depósito mineral de FarimSaliquinhé apresenta morfologia tabular, onde coexistem duas formações fosfáticas FPA e FPB, cobertas por uma espessa camada de materiais heterogêneos, predominantemente inconsolidados, e, designadas, genericamente, de cobertura. Um modelo conceptual simplista da forma coloca essas camadas pela seguinte ordem, da base para o topo: FPB, FPA e cobertura (Figura 2). O rio Cacheu contacta o depósito mineral a Sul.

\section{Metodologia}

Na fase de estudo de viabilidade econômica de um depósito mineral, com potencialidades para exploração mineira, a caracterização e a avaliação de reservas são, normalmente, baseadas em sondagens verticais, desde a superfície até atravessarem o depósito. Idealmente a avaliação de reservas compreende o cálculo das tonelagens de minério, estéril e de teores da variável de interesse econômico.

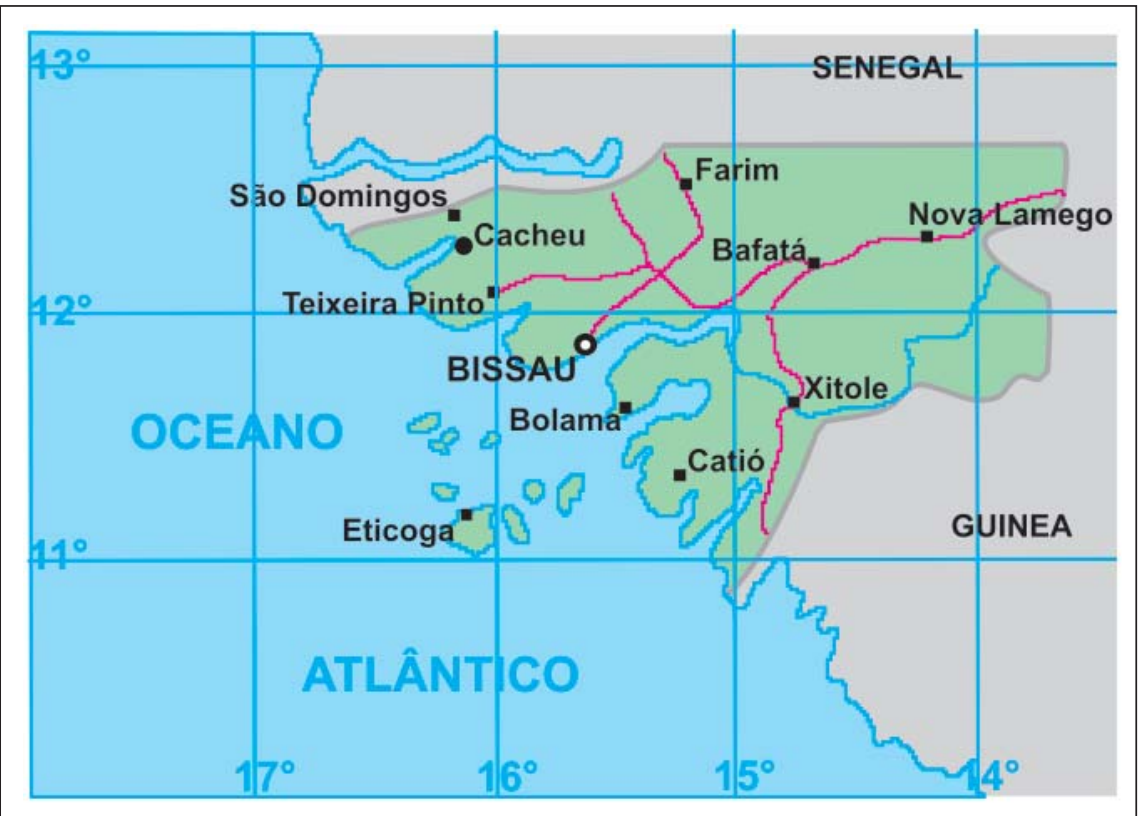

Figura 1 - Localização da povoação de Farim na Guiné Bissau. 
cada sondagem, foram efetuadas uma ou várias medições de teores, a diferentes profundidades, tendo sido registradas a profundidade da base de cada amostra. A diferença entre duas profundidades consecutivas define um suporte.

Todos os teores dessa tabela de partida foram calculados ponderando os valores obtidos nas determinações analíticas com o comprimento do respectivo suporte. Nesse estudo, apenas foram modelados os teores em $\mathrm{P}_{2} \mathrm{O}_{5}$, dado que todas as indicações vão no sentido de não considerar a existência de componentes penalizantes.

A metodologia utilizada, na caracterização e na avaliação das reservas geológicas do depósito mineral de Farim-Saliquinhé, encontra-se sintetizada no diagrama da Figura 3.

A caracterização da forma é, no essencial, a estimação em cada local M, $\mathrm{P}$ (ou X, Y) da espessura da cobertura $[e(x)]$ e possança de FPA $\left[p_{A}(x)\right]$ e FPB $\left[p_{B}(x)\right]$. Para a estimação da espessura de cobertura, considerou-se a krigagem com deriva externa, onde a deriva são as cotas da superfície (relevo). Para as possanças de FPA e FPB, considerou-se a krigagem normal (Soares, 2000).

Para cada localização xu da área de estudo A, com a possança das formações FPA e FPB $\left[p_{A}(x)\right]$ e $\left[p_{B}(x)\right]$, e os respectivos teores em $\mathrm{P}_{2} \mathrm{O}_{5},\left[\mathrm{z}_{A}(x)\right] \mathrm{e}$ $\left[z_{B}(x)\right]$, definem-se as variáveis auxiliares acumulação:

FPA: $a_{A}\left(x_{u}\right)=p_{A}\left(x_{u}\right) \cdot z_{A}\left(x_{u}\right) \mathrm{e}$

FPB: $a_{B}\left(x_{u}\right)=p_{B}\left(x_{u}\right) \cdot z_{B}\left(x_{u}\right)$

Essas variáveis auxiliares representam a quantidade de fosfato $\left(\mathrm{P}_{2} \mathrm{O}_{5}\right)$ na espessura da camada e, por isso, tratam-se de variáveis auxiliares, ao contrário dos teores que dizem respeito a possanças variáveis. São, por isso, expressas em \%.m de $\mathrm{P}_{2} \mathrm{O}_{5}$. A utilização dessas variáveis auxiliares é uma prática comum na modelação desse tipo de depósitos minerais, onde os teores são medidos sobre suportes com dimensão variável, conforme a espessura local da camada amostrada.
No que se refere à incerteza, as reservas foram classificadas com base na simulação geoestatística e em grandes painéis correspondentes a um ano de exploração com o ritmo fixado em 1 milhão de toneladas de fosfato $\left(\mathrm{P}_{2} \mathrm{O}_{5}\right)$. Para cada grande painel, e tendo por base cem realizações da variável acumulação, analisaram-se os histogramas locais e consideram-se as seguintes classes de incerteza com significância de 90\% (JORC, 1999):

I) Reservas medidas - Quando existe pouca incerteza nos teores. Considerou-se, nesse estudo, quando 90\% das realizações simuladas estão num intervalo de $\pm 10 \%$ do teor médio.

II) Reservas indicadas - Quando existe alguma incerteza na caracterização dos teores. Nesse estudo, considerou-se, nessa classe, que $90 \%$ das realizações estão num intervalo de $\pm 20 \%$ em torno do teor médio.

III) Reservas inferidas - Quando a incerteza é ainda mais elevada. Nessa classe, consideraram-se os blocos onde $90 \%$ das realizações estão num intervalo de $\pm 30 \%$ em torno da média.

Essa significância deverá ser confirmada ou reavaliada no futuro com as consequências que terão no rendimento

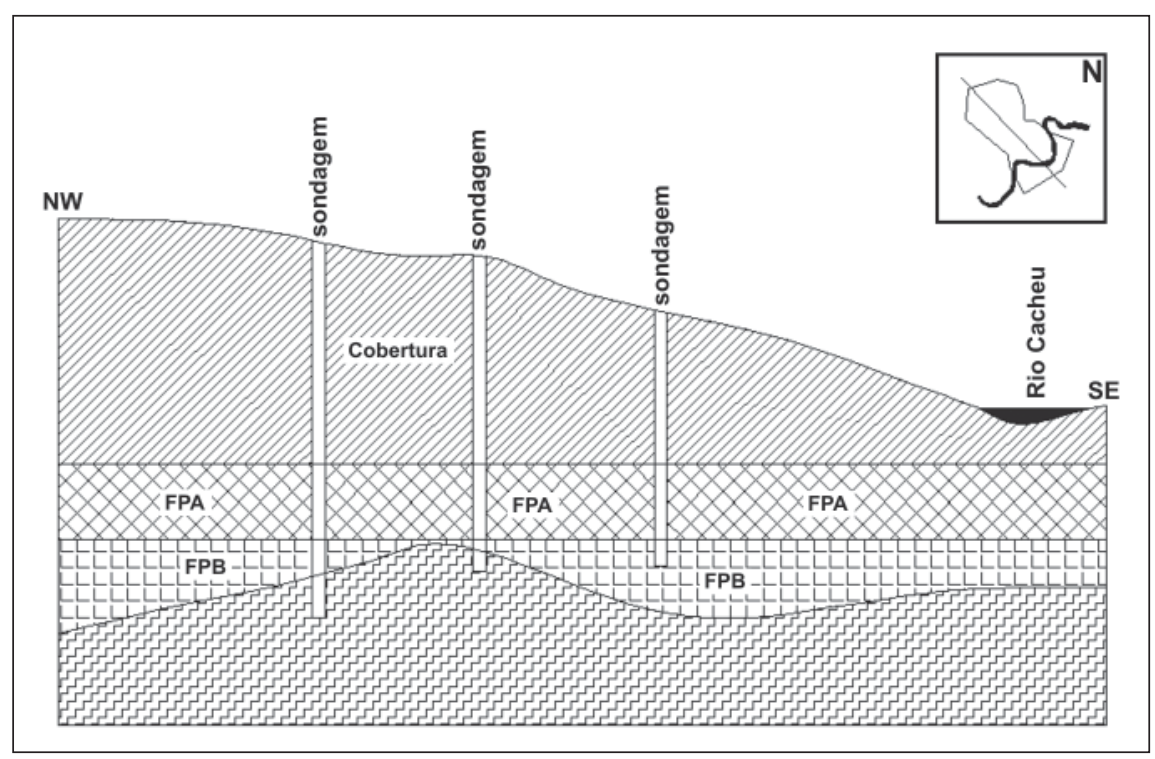

Figura 2 - Modelo conceptual geológico simplificado. da mina de eventuais flutuações nos teores do fosfato, nomeadamente no que se refere ao processo de concentração e dimensionamento de um parque de homogeneização.

\section{Resultados e discussão}

\subsection{Modelação geoestatística do depósito mineral de fosfato de Farim-Saliquinhé}

A caracterização da área em estudo foi feita para uma malha $2 \mathrm{D}$ de blocos, de seção quadrada, com $40 \times 40 \mathrm{~m}$, totalizando 15.819 blocos-seleção. A área em estudo é, assim, de $25.310 .400 \mathrm{~m}^{2}$, ou seja, 2.531,04 ha. Em todas as estimações por krigagem, foram consideradas as vinte amostras vizinhas mais próximas.

\section{Análise uni e bivariada}

Os teores em $\mathrm{P}_{2} \mathrm{O}_{5}$ são mais elevados na formação FPA do que na FPB. Por exemplo, enquanto que, na formação FPB, os maiores teores não excedem os $30 \%$, a mediana dos teores em FPA é de $30,70 \%$, ou seja, cerca de metade da população dos teores de FPA é superior ao máximo observado em FPA (ver Figura 4). 


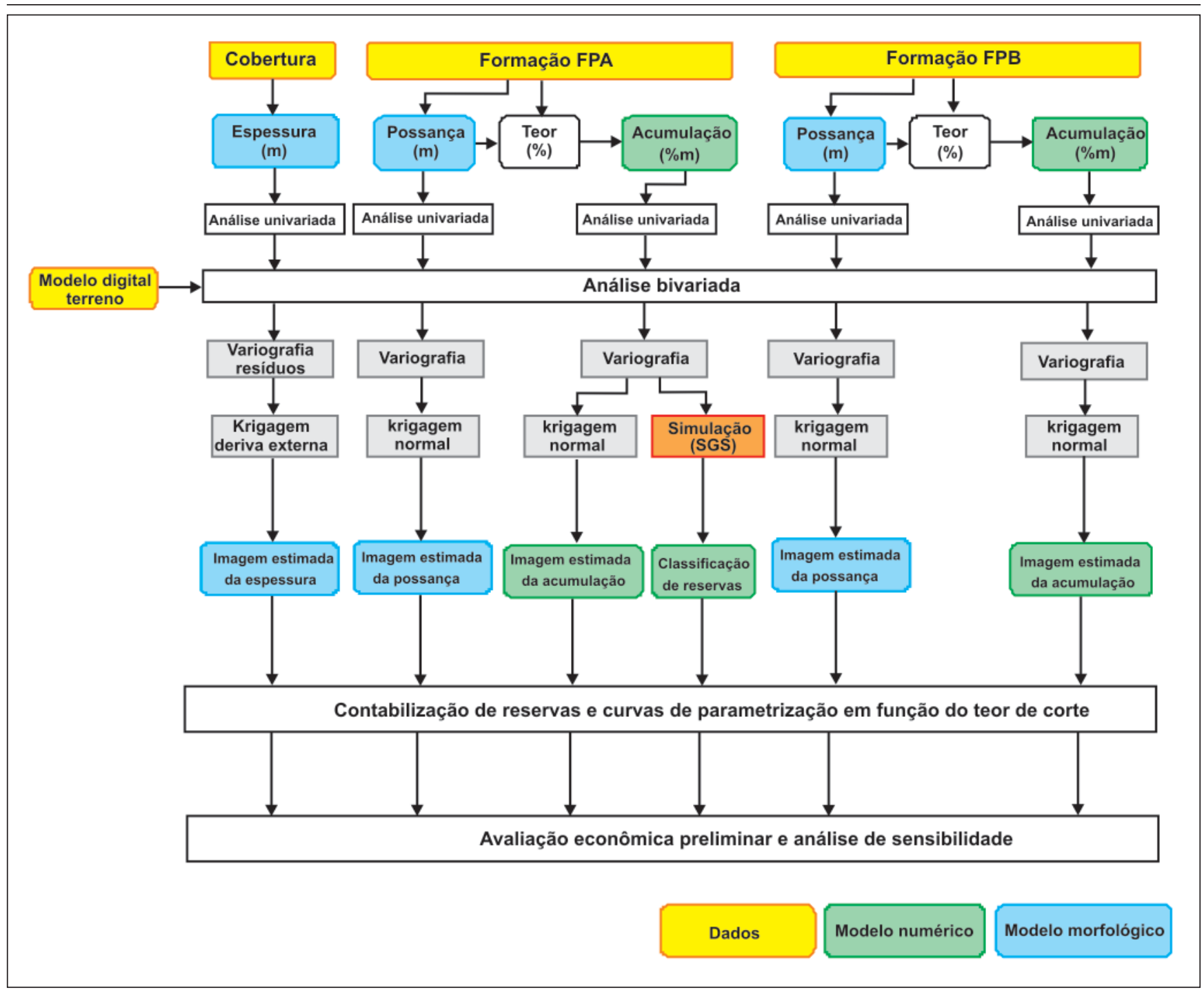

Figura 3 - Diagrama da metodologia proposta para a caracterização e avaliação de reservas do depósito mineral de Farim-Saliquinhé.

Na Tabela 1, apresenta-se a matriz de correlações entre as variáveis morfológicas estudadas, onde se destaca a excelente correlação entre as cotas do terreno e a espessura de cobertura, que fundamentam a utilização da krigagem com deriva externa na estimação da espessura de cobertura, utilizando como informação secundária o modelo digital da superfície construído com o levantamento topográfico do BRGM. Observa-se, ainda, correlação moderada e negativa entre a possança de FPA e a de FPB, que é porventura proveniente da indefinição da transição das duas formações, ou seja, quando se faz a classificação da amostra para FPB, retira-se a metragem de FPA e vice-versa.
Na Tabela 2, apresenta-se a matriz de correlações entre a possança e a acumulação nas formações FPA e FPB. Destaca-se a elevada correlação entre a possança e a acumulação sempre que consideradas da mesma formação (0,97 e 0,95 respectivamente para FPA e FPB). Cabe destacar que a acumulação deriva do produto da possança pelo teor, mas uma correlação elevada evidencia que os teores são concordantes. Da mesma forma, as correlações negativas entre a possança e a acumulação de formações diferentes relevam a correlação negativa entre a possança de FPA e de FPB.

\section{Modelo morfológico}

Os variogramas das variáveis espessura de cobertura (em termos de resíduos) e possança de FPA e FPB foram ajustados com modelos isótropos, de tipo esférico, sem efeito de pepita e com 2000 m, 1600 m e 1750 m, respectivamente (Figura 5).

Os resultados das estimações da superfície, da espessura de cobertura e da espessura das formações FPA e FPB podem ser visualizados na representação 3D da Figura 6. 
Gilberto Charifo et al.

\section{FPA (m)}

Média: $\mathbf{2 9 , 1 8 \%}$

Mediana: $\mathbf{3 0 , 7 0 \%}$

Desvio-padrão: $6,06 \%$

Variân cia: $36,72 \%^{2}$

Coef. skewness: $-1,84$

Amplitude: $33,30 \%$

Minimo: $3,30 \%$

Máximo: $36,60 \%$

$\mathrm{N}^{\circ}$ de amostras: 144

\section{FPB (m)}

Média: $14,53 \%$

Mediana: $14,20 \%$

Desvio-padrão: $4,86 \%$

Variância: $23,65 \%^{2}$

Coef. skewness: 0,26

Amplitude: $23,30 \%$

Minimo: $4,50 \%$

Máximo: $27,80 \%$

$\mathrm{N}^{\circ}$ de amostras: 91

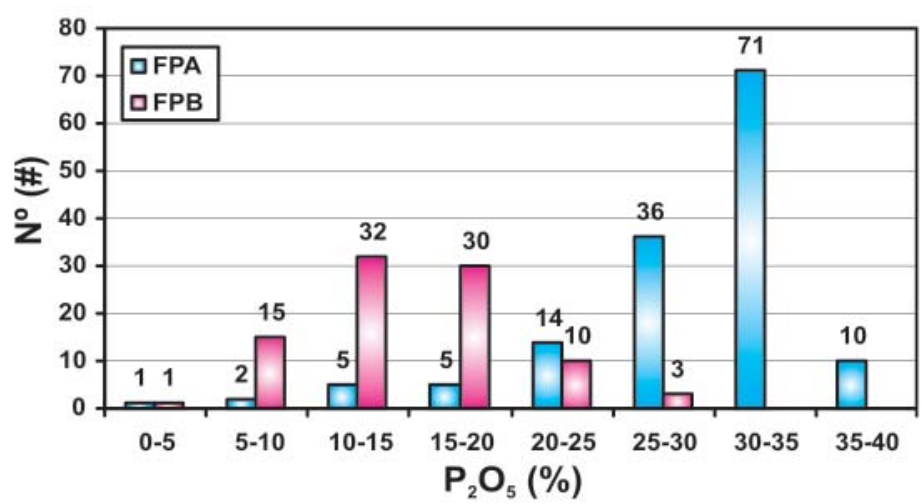

Figura 4 - Estatísticos básicos, histograma e box-plot dos teores em $\mathrm{P}_{2} \mathrm{O}_{5}$ de FPA e FPB.

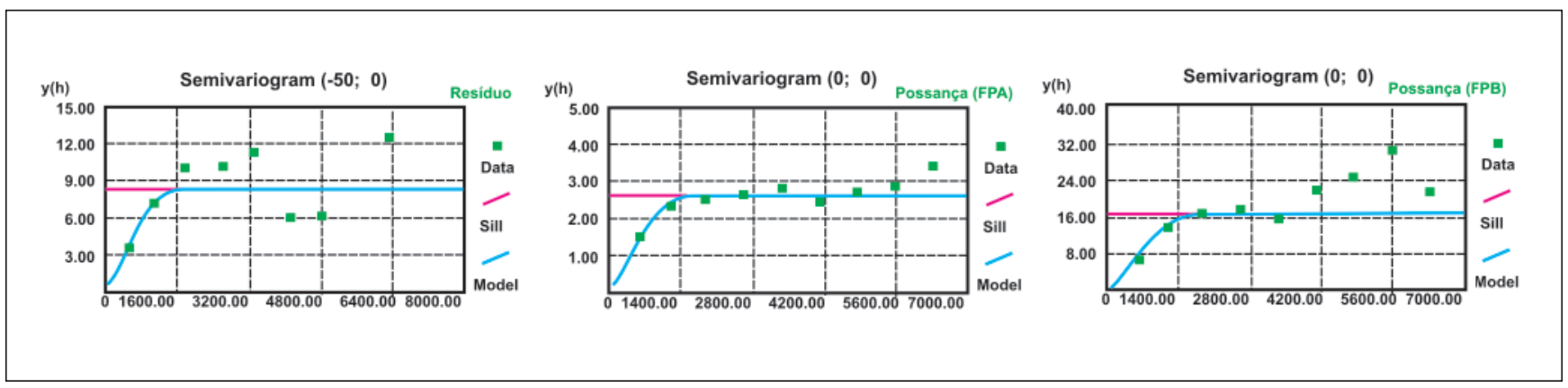

Figura 5 - Variogramas da variável acumulação de FPA (esquerda) e FPB (direita).

\section{Modelo de teores}

O modelo de teores é o modelo da variável acumulação para as formações FPA e FPB. Os variogramas das variáveis acumulação de FPA e de FPB foram ajustados com modelos isótropos, de tipo esférico, sem efeito de pepita e com amplitude de 1600 e 2500 metros, respectivamente (Figura 7). Na Figura 8 , ilustram-se os resultados obtidos por krigagem.

\section{Apresentação das reservas}

Os resultados das reservas apresentam-se na Tabela 3 para 4 teores de corte. Esses dados permitem dizer que as reservas totais prováveis são de 115.684.300 toneladas de minério de fosfato FPA, com teor médio de 30,81\%, correspondendo a 35.637.156 toneladas de fosfato.

\section{Classificação das reservas}

Para apresentar as reservas por classes de incerteza, geraram-se cem imagens simuladas da variável acumulação para a formação FPA.
Tabela 1 - Matriz de correlação das variáveis morfológicas estudadas.

\begin{tabular}{c|c|c|c|c}
\hline & Cota & Cobertura & Possança FPA & Possança FPB \\
\hline Cota & 1,00 & 0,96 & 0,14 & 0,17 \\
\hline Cobertura & & 1,00 & 0,08 & 0,20 \\
\hline Possança FPA & & & 1,00 & $-0,60$ \\
\hline Possança FPB & & & & 1,00 \\
\hline
\end{tabular}

Tabela 2 - Matriz de correlação entre a possança e a acumulação de FPA e FPB.

\begin{tabular}{l|c|c}
\hline & Possança FPA & Possança FPB \\
\hline Acumulação FPA & 0,97 & $-0,60$ \\
\hline Acumulação FPB & $-0,65$ & 0,95 \\
\hline
\end{tabular}

Se considerarmos o objetivo de produção de minérios para perfazer anualmente 1.000.000 ton de $\mathrm{P}_{2} \mathrm{O}_{5}$, verifica-se que cada bloco-seleção, que serviu de suporte às estimações, com 40 por 40 metros, é explorado em média a cada 1,5 dias. De forma a tornar a classificação menos restrita, optou-se por analisar a classificação das reservas para grandes painéis, correspondentes a um ano de exploração (em média mais de 500 blocos-seleção). 


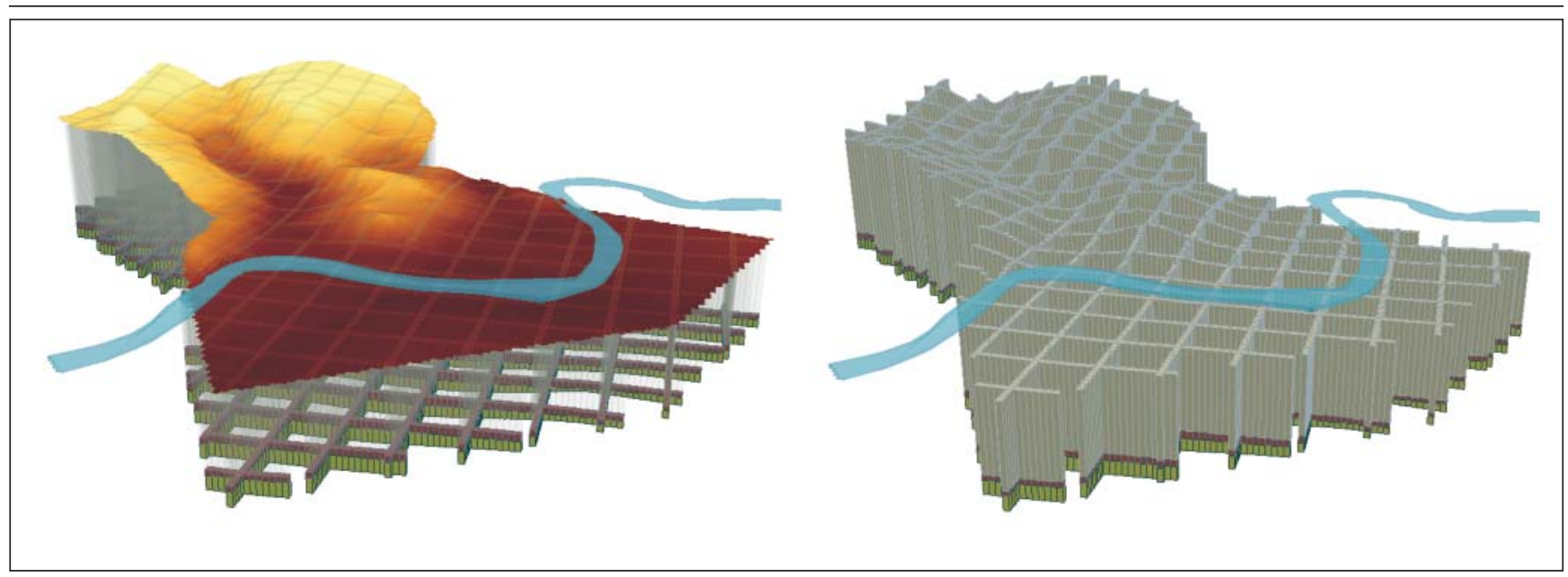

Figura 6 - Visualização 3D da superfície, cobertura, e das formações FPA e FPB.

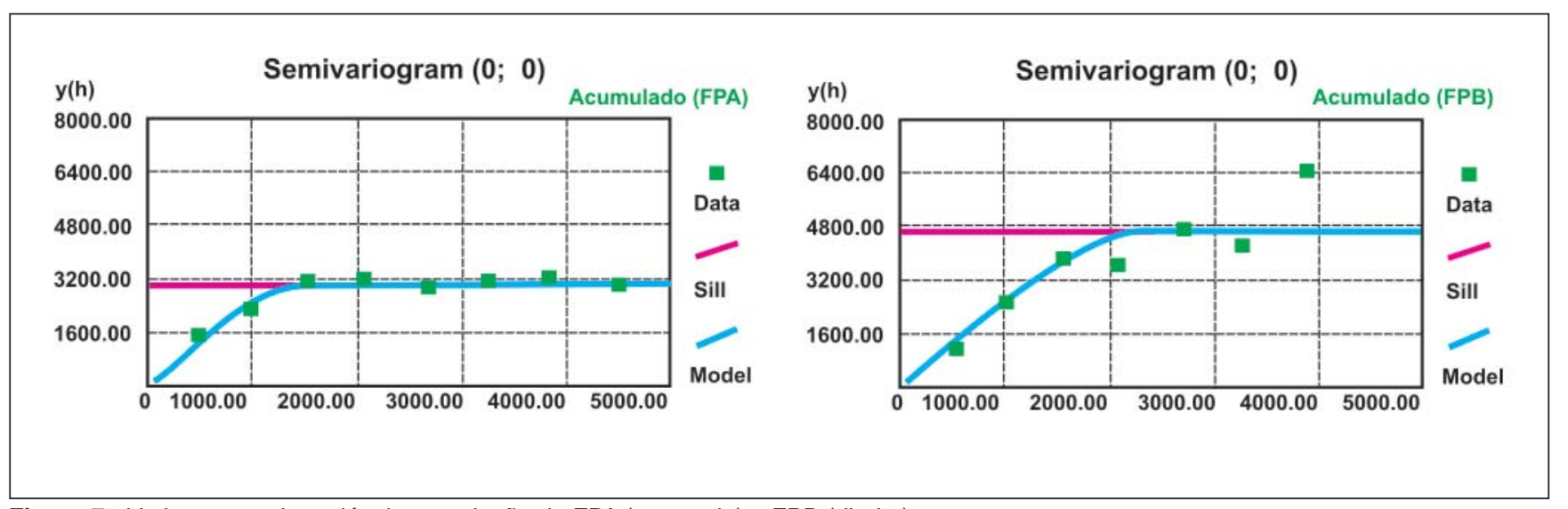

Figura 7 - Variogramas da variável acumulação de FPA (esquerda) e FPB (direita).

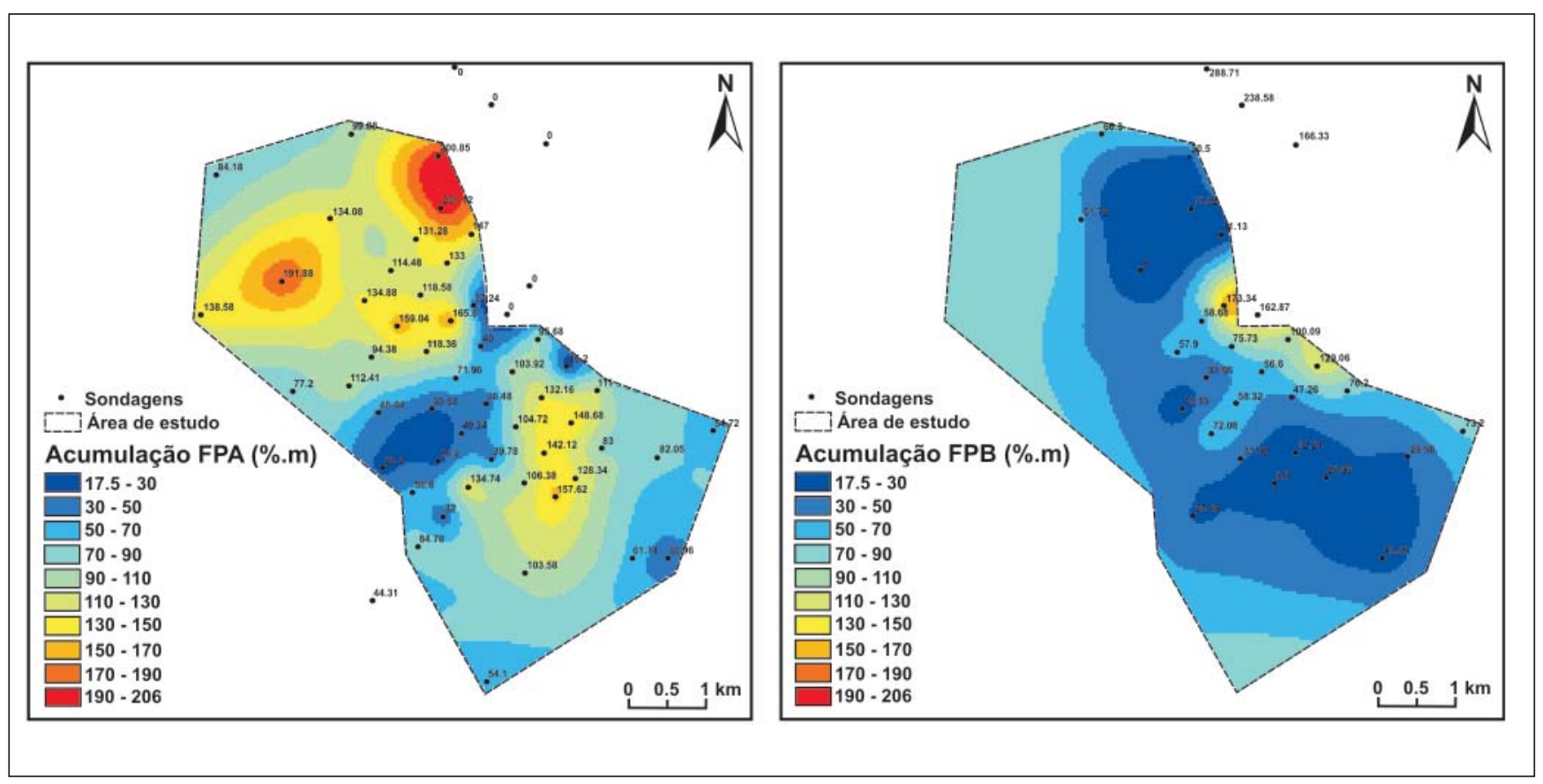

Figura 8 - Imagens estimadas da acumulação: FPA (esquerda) e FPB (direita).

574 REM: R. Esc. Minas, Ouro Preto, 63(3): 569-580, jul. set. 2010 
Gilberto Charifo et al.

Tabela 3 - Síntese de reservas da massa mineral de Farim-Saliquinhé.

\begin{tabular}{|c|c|c|c|c|c|}
\hline \multirow{2}{*}{\multicolumn{2}{|c|}{ Descritor }} & \multicolumn{4}{|c|}{ Teor de corte em $\mathrm{P}_{2} \mathrm{O}_{5}$ na unidade FPA } \\
\hline & & $\begin{array}{l}6,62 \% \\
(\min )\end{array}$ & $\begin{array}{c}27,5 \% \\
\text { (percentil 20\%) }\end{array}$ & $\begin{array}{c}30 \% \\
\text { (percentil 41\%) }\end{array}$ & $\begin{array}{c}32,5 \% \\
\text { (percentil } 78 \% \text { ) }\end{array}$ \\
\hline & Área $\left(\mathrm{m}^{2}\right)$ & 25.310 .400 & 20.395 .200 & 14.980 .800 & 5.772 .800 \\
\hline \multirow{2}{*}{ Estéril } & Volume $\left(\mathrm{m}^{3}\right)$ & 991.864 .800 & 802.932 .500 & 607.784 .900 & 256.146 .000 \\
\hline & Espessura média (m) & 39,19 & 39,37 & 40,57 & 44,37 \\
\hline \multirow{5}{*}{ FPA } & Q. minério (tons) & 115.684 .300 & 97.276 .160 & 75.275 .730 & 31.076 .020 \\
\hline & Q. fosfato (tons) & 35.637 .156 & 31.187 .225 & 24.825 .669 & 11.030 .752 \\
\hline & Possança média $(\mathrm{m})$ & 3,20 & 3,34 & 3,51 & 3,76 \\
\hline & REM & 12,26 & 11,8 & 11,55 & 11,79 \\
\hline & Teor médio (\%) & 30,81 & 32,06 & 32,98 & 35,50 \\
\hline \multirow{5}{*}{ FPB } & Q. minério (tons) & 145.296 .800 & 119.214 .100 & 90.075 .000 & $41,772,630$ \\
\hline & Q. fosfato (tons) & 19.163 .719 & 15.294 .979 & 11.052 .785 & 4.438 .018 \\
\hline & Possança média (m) & 3,83 & 3,90 & 4,00 & 4,82 \\
\hline & REM & 10,24 & 10,10 & 10,12 & 9,20 \\
\hline & Teor médio (\%) & 13,19 & 12,83 & 12,27 & 10,62 \\
\hline
\end{tabular}

Os painéis foram construídos a partir de fiadas de blocos-seleção, no sentido este-oeste, e avançando de sul para norte. Ao todo foram desenhados 30 painéis correspondentes a 30 anos de exploração, mais um painel (31) com a área remanescente (Figura 9).

Para os painéis de exploração referidos, correspondentes a um ano de exploração, pode-se caracterizar as reservas, predominantemente, como inferidas.

\subsection{Avaliação econômica preliminar do projeto de exploração}

Para essa análise, assume-se que a exploração será feita a céu aberto, por decapagem da superfície até ao nível freático do local (que se sabe ser próximo da superfície) e dragagem da restante fração. Assume-se, ainda, que o minério não é concentrado no local

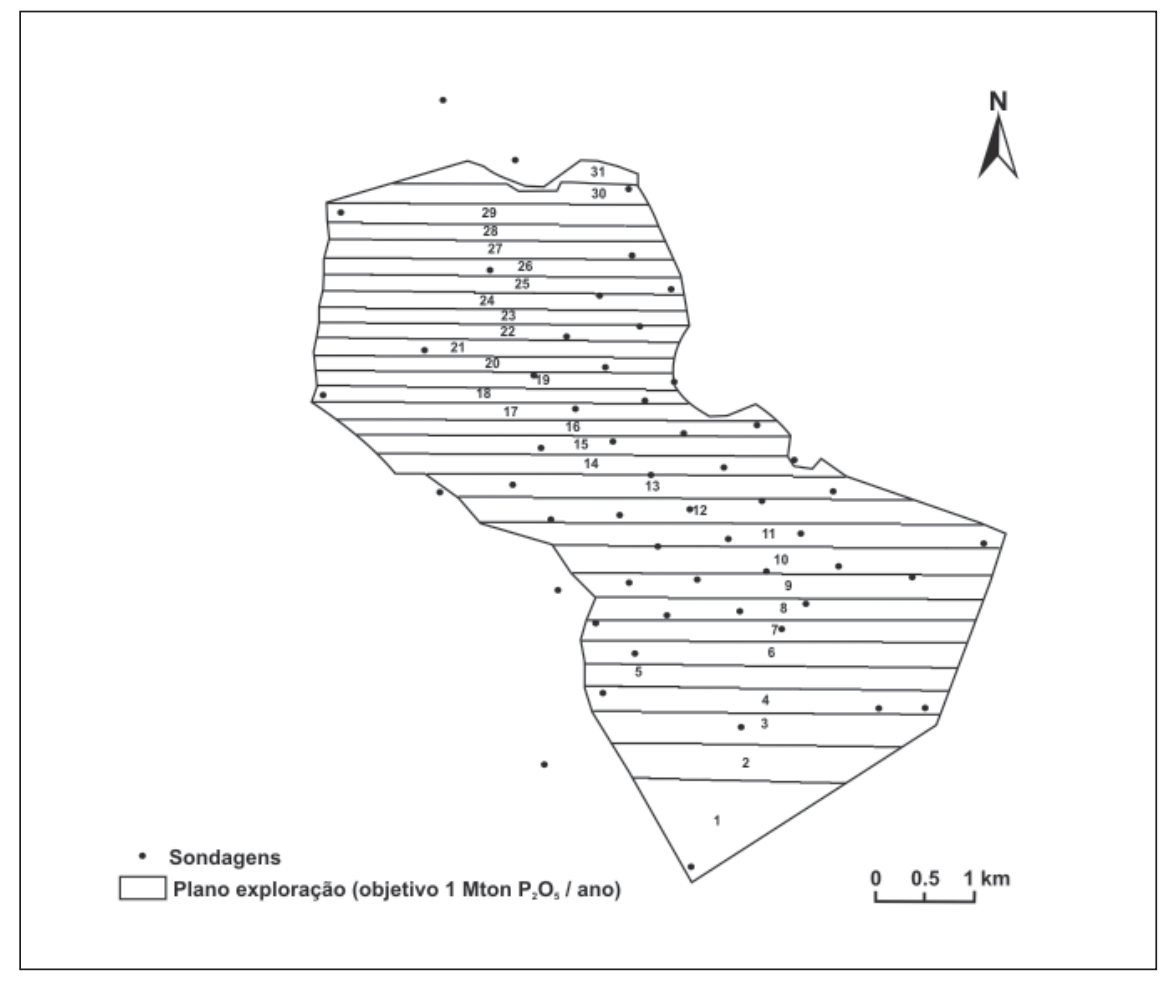

Figura 9 - Plano-painéis de exploração com objetivo fixado a 1.000 .000 ton de $\mathrm{P}_{2} \mathrm{O}_{5}$ por ano. 
de exploração, mas é transportado por barco, implicando custo de transporte, para uma lavaria a $2000 \mathrm{~km}$, onde é concentrado. O custo de tratamento é descontado no preço de venda como uma fracção percentual.

Na avaliação econômica, consideraram-se duas etapas:

I) A primeira, onde é contabilizado o benefício por bloco, ou unidadeseleção, sem assumir os custos de investimento e o ritmo de exploração.

II) Uma etapa posterior, onde se assume um investimento inicial e onde se projectam os Movimentos de Caixa para o período de exploração e estimam a taxa interna de retorno (TIR) do investimento.

Nessa avaliação, assumiram-se os seguintes preços e custos num cenáriobase e indicam-se os parâmetros onde se fez a análise de sensibilidade (Tabela 4).

Na Figura 10, apresenta-se a variação dos benefícios globais por taxa de variação dos parâmetros analisados.

Relativamente aos dois parâmetros de custo, o gráfico mostra que o custo de extracção é mais sensível comparativamente ao custo de transporte. No que respeita ao preço de venda, o benefício é bastante sensível a esse parâmetro (maior declive da reta). Considera-se que a alteração do preço de venda e o desconto por não concentração têm o mesmo universo de respostas e, por isso, as duas representações aparecem sobrepostas.

Na Figura 11, mostram-se os blocos-seleção que devem ser explorados, com benefícios positivos, conforme cada um dos quatro cenários de variação do preço de venda por tonelada (US\$24, US\$30, US\$50 e US\$60), mais o Cenário-Base (US\$40). As áreas a explorar correspondem, da esquerda para a direita, a 34,53; 67,78; 91,18; 96,76 e 99,52\% da área total.

Para elaborar os mapas de fluxo de caixa, numa base temporal anual, e apenas para o Cenário-Base, foram construídas duas hipotéticas sequências de exploração com avanço de sul para

Tabela 4 - Estrutura de preços e custos no Cenário Base.

\begin{tabular}{c|c|c}
\hline & Valor & $\begin{array}{c}\text { Análise } \\
\text { sensibilidade }\end{array}$ \\
\hline Custo de extração & $1,20 \$ / \mathrm{m}^{3}$ & Sim \\
\hline Custo de transporte por barco para a lavaria & $0,002 \$ / \mathrm{km}$ & Sim \\
\hline Distância entre a mina e a lavaria & $2.000 \mathrm{~km}$ & Não \\
\hline Preço venda do minério de fosfato & $40 \$ /$ ton & Sim \\
\hline Desconto preço venda para concentração & $35 \%$ & Sim \\
\hline
\end{tabular}

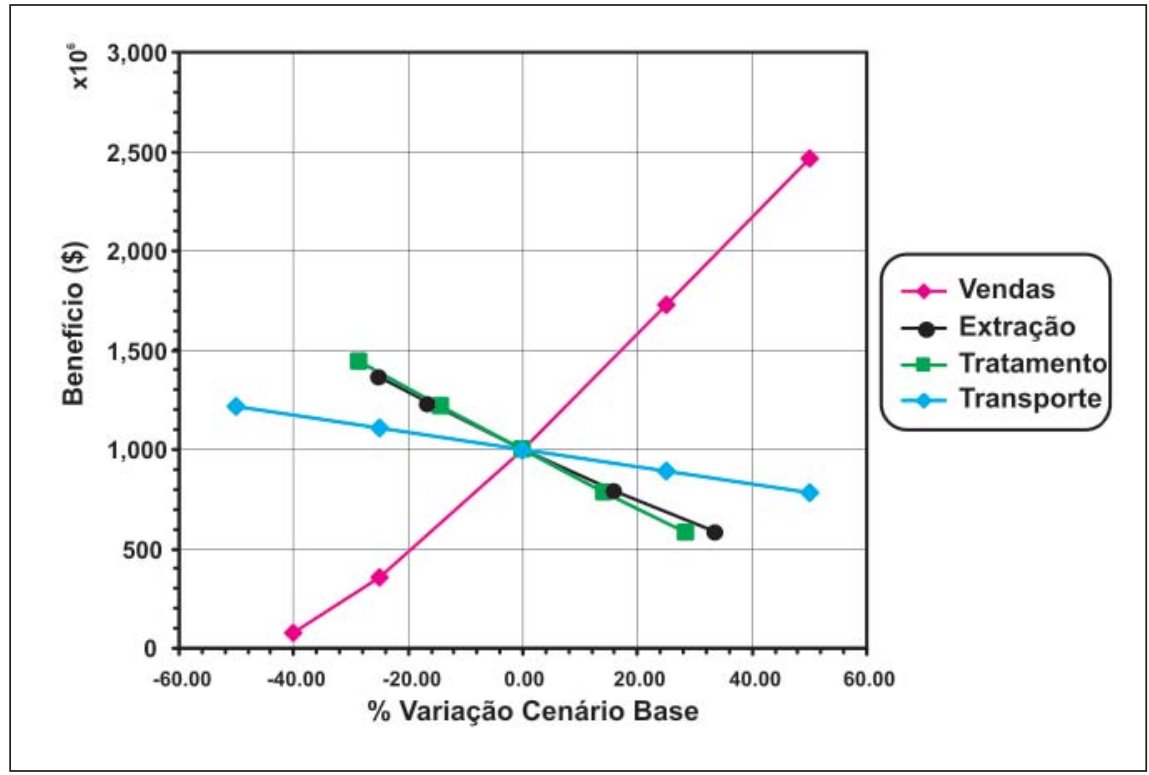

Figura 10 - Análise de sensibilidade do benefício ao preço de venda e custos de extracção, transporte e tratamento.

norte (Figura 5) ou de norte para sul, segundo fatias com desenvolvimento este-oeste com 40 metros de largura. No que se refere ao ritmo de exploração, foi definido o mesmo objetivo anual já utilizado para a classificação das reservas, ou seja, a exploração anual de 1.000 .000 toneladas de $\mathrm{P}_{2} \mathrm{O}_{5}$, o que perfaz a exploração ao longo de trinta anos completos mais quatro meses. Os pressupostos assumidos estão sintetizados na Tabela 5.

Os resultados ano a ano do volume de cobertura $\left(\mathrm{m}^{3}\right)$, a quantidade de minério FPA (ton), os benefícios (\$/ano), os custos de extração e o tratamento (\$/ano), o teor médio anual $\left(\% \mathrm{P}_{2} \mathrm{O}_{5}\right)$ e a relação estéril-minério - REM, para os cenários N-S e S-N, encontram-se representados, graficamente, na Figura 12.
Nesses gráficos, destaca-se que a exploração, no sentido S-N, é, aparentemente, mais vantajosa, dado que retorna maiores benefícios nos primeiros anos. Esse fato é, principalmente, derivado da menor espessura de cobertura na parte sul, que aumenta para norte. No que se refere aos teores, existe uma ligeira tendência de subida no longo prazo na exploração de $\mathrm{S}-\mathrm{N}$, mas que não é compensada com o aumento da espessura de cobertura na contabilidade dos benefícios.

Os mapas de fluxo de caixa (na Tabela 6 mostra-se o mapa referente à hipotética exploração no sentido S-N) mostram que a taxa interna de retorno (TIR) do projeto de exploração S-N é de $17,72 \%$, enquanto que, para o projeto 


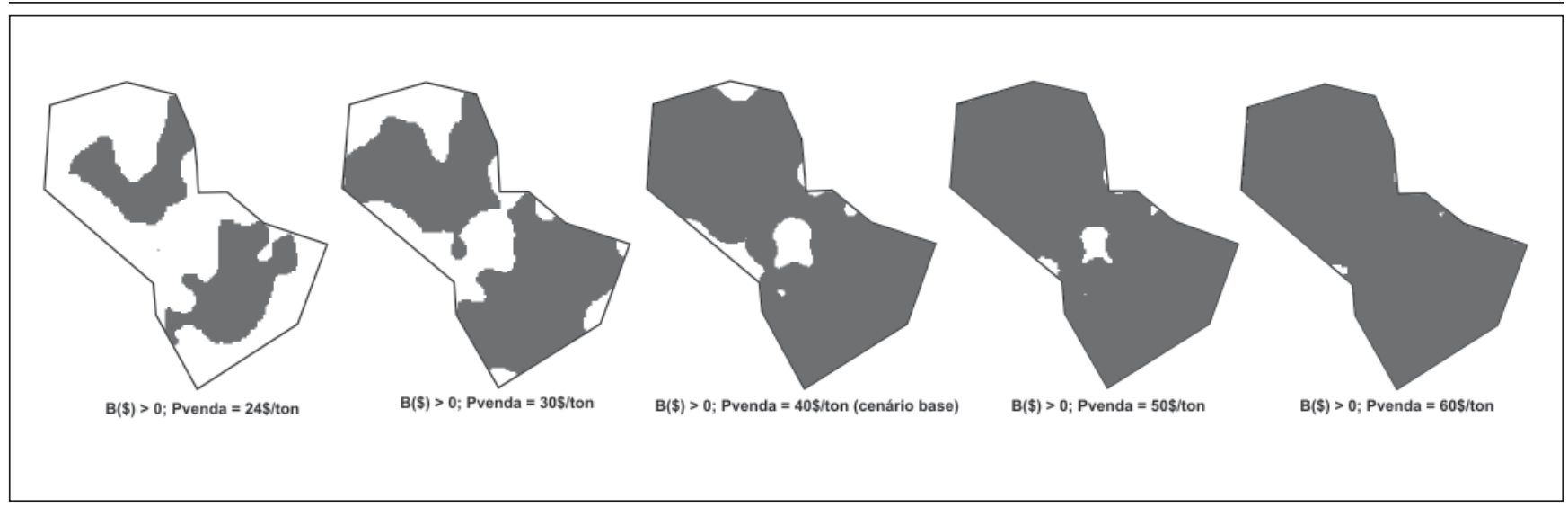

Figura 11 - Blocos-seleção com benefício positivo, cenário-base mais cenários de variação do preço de venda.

N-S, é de 13,21\%, significativamente, inferior.

Para se ter uma noção da influência do investimento inicial, apresentam-se os gráficos da Figura 13, onde se mostra que uma redução no investimento inicial de $150 \mathrm{M} \$$ para $100 \mathrm{M} \$$ aumentaria a TIR para cerca de $25 \%$, que são, sem dúvida, valores mais razoáveis para um projeto mineiro com elevado risco associado.

\section{Conclusões}

De acordo com trabalho realizado, podem ser retiradas as seguintes conclusões:
Tabela 5 - Pressupostos para exploração do depósito mineral de Farim-Saliquinhé.

\begin{tabular}{c|c}
\hline & Valor \\
\hline Investimento inicial & 150 Milhões \$ \\
\hline Ritmo de exploração & 1.000 .000 ton fosfato $\mathrm{P}_{2} \mathrm{O}_{5}$ \\
\hline Depreciação & Linear no tempo \\
\hline Taxa sobre lucros & $30 \%$ \\
\hline Taxa de inflação & Não aplicável \\
\hline Taxas ou fundos ambientais & \\
\hline
\end{tabular}

Tabela 6 - Mapa de fluxos de caixa (cenário exploração S-N).

\begin{tabular}{c|c|c|c|c|c|c|c}
\hline Anos & $\mathbf{0}$ & $\mathbf{1}$ & $\mathbf{2}$ & $\mathbf{3}$ & $\mathbf{4}$ & $\ldots$ & 30 \\
\hline Vendas (\$) & & $96,160,820$ & $99,405,790$ & $98,112,970$ & $95,717,300$ & $84,887,310$ & 38118700 \\
\hline Custos operacionais (\$) & & $-65,904,770$ & $-60,923,300$ & $-59,588,250$ & $-56,555,500$ & $-69,249,570$ & -32689780 \\
\hline Depreciação (\$) & & $-4,838,710$ & $-4,838,710$ & $-4,838,710$ & $-4,838,710$ & $-4,838,710$ & $-4,838,710$ \\
\hline Valor antes taxas (\$) & & $25,417,340$ & $33,643,780$ & $33,686,010$ & $34,323,090$ & $10,799,030$ & 590,21 \\
\hline Taxas (30\%) & & $-7,625,202$ & $-10,093,134$ & $-10,105,803$ & $-10,296,927$ & $-3,239,709$ & $-177,063$ \\
\hline Lucros (\$) & & $17,792,138$ & $23,550,646$ & $23,580,207$ & $24,026,163$ & $7,559,321$ & 413,147 \\
\hline Mais depreciação (\$) & & $4,838,710$ & $4,838,710$ & $4,838,710$ & $4,838,710$ & $4,838,710$ & $4,838,710$ \\
\hline Custos de capital (\$) & -150.000 .000 & & & & & & \\
\hline Fluxo de caixa (\$) & -150.000 .000 & $22,630,848$ & $28,389,356$ & $28,418,917$ & $28,864,873$ & $12,398,031$ & $5,251,857$ \\
\hline
\end{tabular}




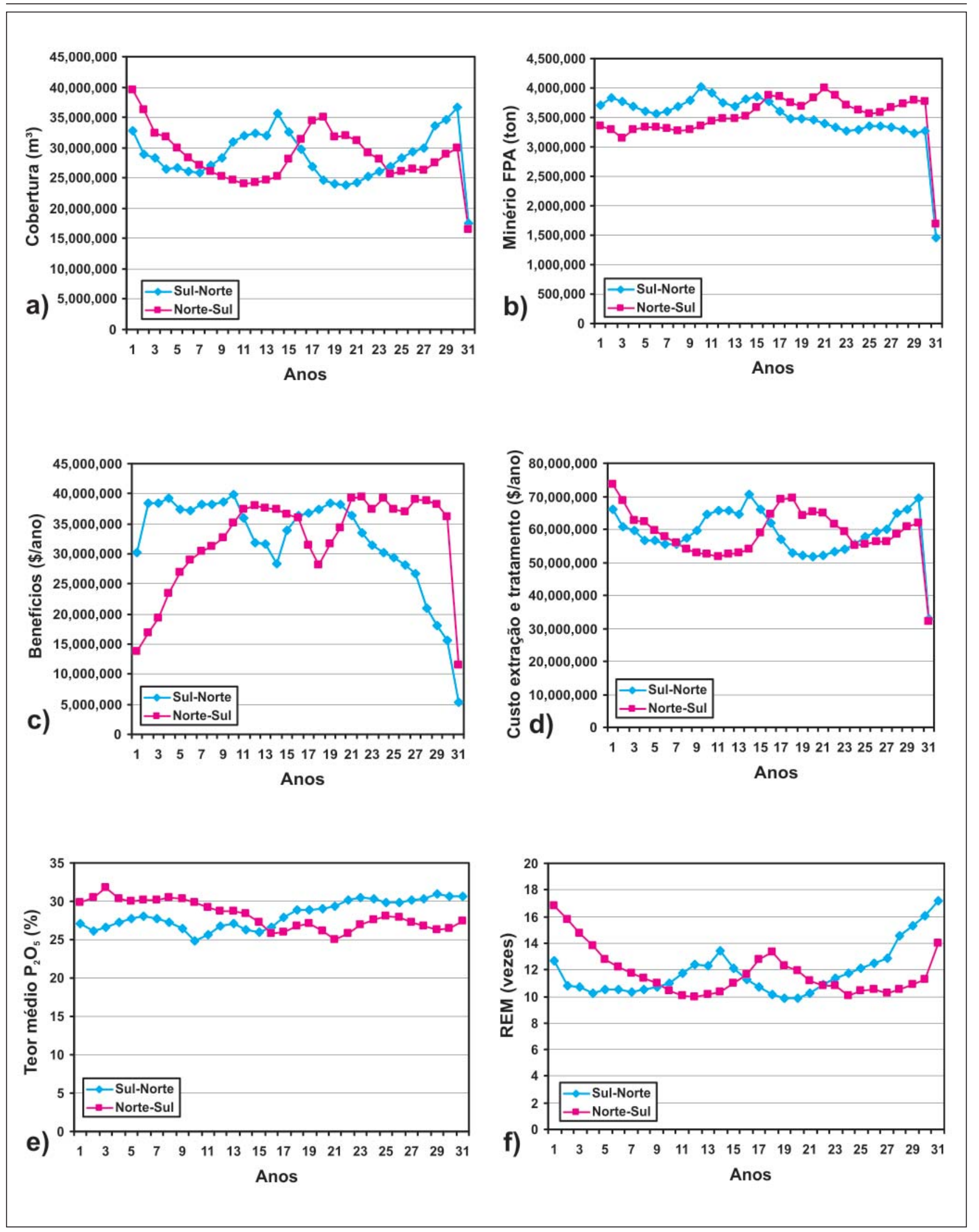

Figura 12 - Gráficos de variação anual de: a) volume de cobertura $\left(\mathrm{m}^{3}\right)$; b) minério FPA (ton); c) benefícios (\$/ano); d) custos de extracção e tratamento (\$/ano); e) teor médio anual $\left.\left(\% \mathrm{P}_{2} \mathrm{O}_{5}\right) ; \mathbf{f}\right) \mathrm{REM}$.

578 REM: R. Esc. Minas, Ouro Preto, 63(3): 569-580, jul. set. 2010 
1) De acordo com estimativas da International Fertilizer Industry Association - IFA, a procura mundial por fertilizantes fosfatados cresceu 3,8\% naquele ano. Ainda segundo a IFA, a capacidade de produção de ácido fosfórico, em 2007, foi estimada em 43 Mt, com 1,3 Mt acima do consumo global e da produção de produtos intermediários (MAP, DAP e TSP) e cresceu 5\% sobre 2006, para 25,3 Mt em $\mathrm{P}_{2} \mathrm{O}_{5}$. As reservas mundiais são concentradas: três países detêm $75 \%$ do total, sendo que $42,3 \%$ encontram-se em Marrocos, que possui 21.000 biliões de toneladas. A China, segundo lugar, possui $26 \%$ e os Estados Unidos, 7\%. O Brasil conta com reservas de 319 milhões de toneladas e a Guiné-Bissau conta com cerca de $120 \mathrm{Mt}$.

2) O depósito mineral de FarimSaliquinhé é de origem sedimentar, com morfologia tabular, onde coexistem duas formações fosfáticas FPA e FPB, cobertas por uma espessa camada de materiais heterogêneos, predominantemente inconsolidados, designados, genericamente, de cobertura. Um modelo conceptual simplista da forma coloca essas camadas pela seguinte ordem, da base para o topo: FPB, FPA e cobertura.

3) Para a área estudada, a tonelagem de minério é de, aproximadamente, 113 milhões de toneladas de FPA, com o teor médio de $30 \%$ em $\mathrm{P}_{2} \mathrm{O}_{5}$, para uma área de $24 \mathrm{~km}^{2}$, correspondendo ao volume total de 79 milhões de $\mathrm{m}^{3}$. A densidade média estimada para o minério FPA foi de 1,43. Devido à boa qualidade de FPA, essa matéria-prima pode ser concentrada por flutuação, um processo menos dispendioso do que a calcinação recomendada para a rocha carbonatofosfática da formação FPB.

4) A formação FPB constitui uma importante reserva de fosfato de baixo teor (10-15\% de $\left.\mathrm{P}_{2} \mathrm{O}_{5}\right)$, com elevado conteúdo em carbonato (relação $\mathrm{CaO} / \mathrm{P}_{2} \mathrm{O}_{5}$ entre 2,9 e 3,9). Considerando a espessura média de 3 m numa área de 100 km², estima-se

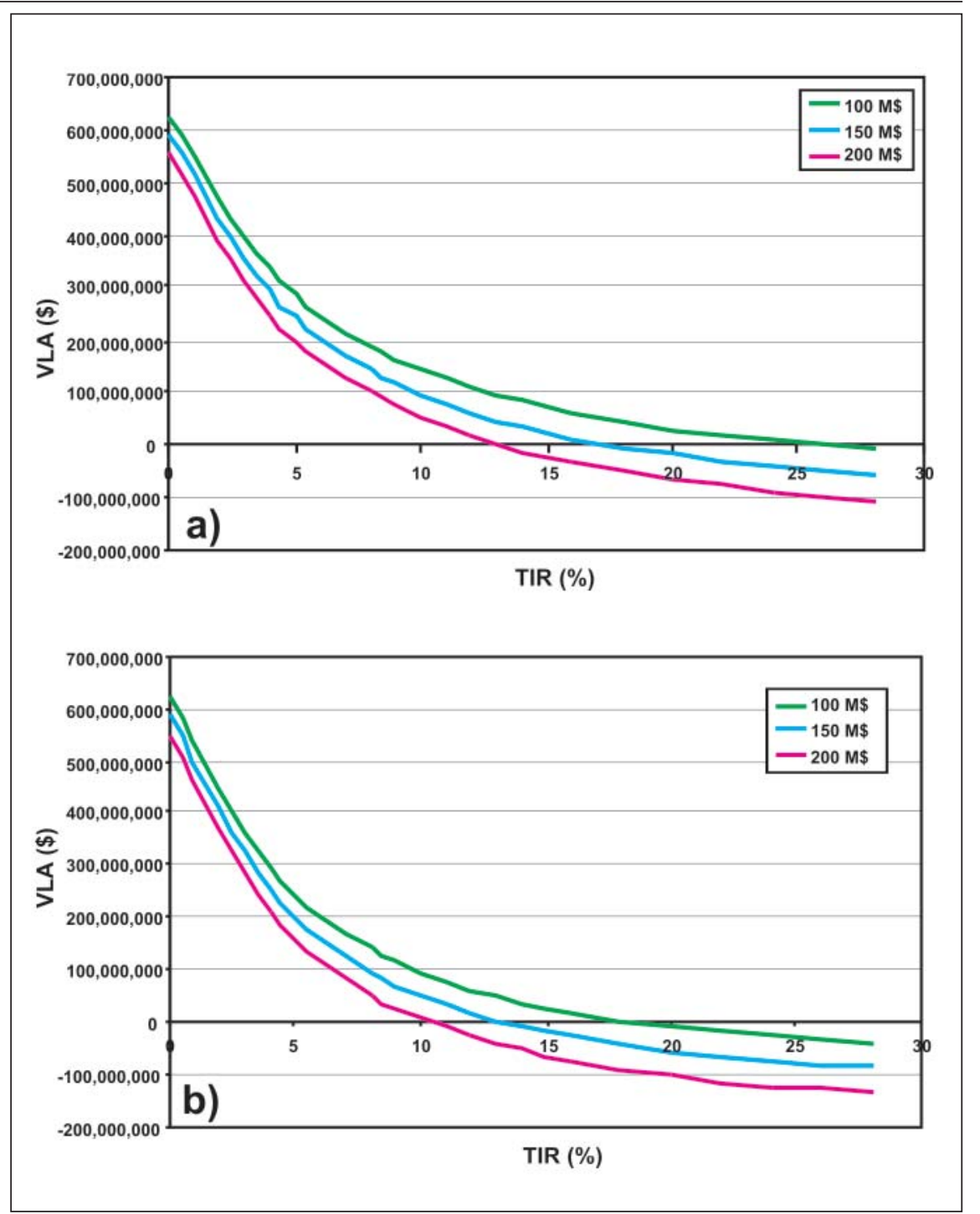

Figura 13 - Curvas de valor líquido atual para três níveis de investimento inicial vs taxa de juro. (a) Exploração N-S. (b) Exploração S-N).

que os quantitativos em minério fosfatado e carbonatado sejam da ordem das 450 milhões de toneladas com teores entre $10-15 \% \mathrm{P}_{2} \mathrm{O}_{5}$.

5) A formulação do modelo de caracterização e de avaliação de reservas geológicas foi desenvolvida nas seguintes etapas: a) Estimação da morfologia do depósito mineral; b) Estimação dos teores em $\mathrm{P}_{2} \mathrm{O}_{5}$ nas formações FPA e FPB; c) Classificação das reservas em inferidas, medidas e indicadas, em função da incerteza local.

6) As reservas totais prováveis, obtidas com o modelo geoestatístico, são de 115.684.300 toneladas de minério na formação FPA, com teor médio de 30,81\%, correspondendo a 35.637.156 toneladas de fosfato. Essas reservas são classificadas quanto à incerteza em inferidas. Os valores agora obtidos são muito semelhantes aos obtidos anteriormente pelo BRGM, aproximadamente 113 milhões de toneladas de FPA com o teor médio de $30 \%$ em $\mathrm{P}_{2} \mathrm{O}_{5}$, correspondendo ao volume total de 79 milhões de $\mathrm{m}^{3}$. A principal vantagem do modelo geoestatístico apresentado é a de possibilitar a confecção de estimativas locais numa malha regular de blocos de seleção, em vez de uma rede de polígonos delimitados pelas sondagens. Também a krigagem é o melhor estimador linear não enviezado. 
7) A vida útil desse depósito mineral é de trinta anos para uma produção estimada de 1.000.000 ton/ano de minério FPA. As curvas de parametrização de reservas das formações FPA e FPB, em função do teor de corte, permitem retirar as seguintes ilações:

- Os teores mais elevados em $\mathrm{P}_{2} \mathrm{O}_{5}$ encontram-se em zonas onde as REM são também mais elevadas. Para teores de corte interiores com cerca de 33\%, a REM estabiliza em torno de 12 vezes.

- A quantidade de minério é sempre superior na formação FPB, relativamente a FPA, mas, no que respeita à quantidade de fosfato, é sempre inferior, cerca de metade para quase todos os teores de corte.

8) A avaliação econômica preliminar do projecto permite esclarecer que a exploração é mais rentável com um cenário de desmonte do sentido S-N, onde a TIR é de 17,72\%. Esse valor pode ser, significativamente, aumentado, se o investimento inicial for reduzido.

\section{Referências bibliográficas}

ALMEIDA, J., SOARES, A. O., ALBUQUERQUE, A. Zonal control to estimate classes of histograms: application to a diamond alluvial deposit. In: BAAFI, E. Y., SCHOFIELD,
N. A. (ed.)., Geostatistics Wollongong'96. Dordrecht: Kluwer Academic Pub., 1997. 2, p. 658-669.

BRGM. Géologie et reserves du gisement de phosphate de chaux de Farim-Saliquinhe (Guinée-Bissau), v.1, 83DAK004, 1983, $51 \mathrm{p}$.

GLACKEN, I. M., SNOWDEN, D. V. Mineral resource estimation, in mineral resource and ore reserve estimation - the AusIMM Guide to good practice. In: EDWARDS, A. C. (Ed.). 2001. p. 189-198 (The Australasian Institute of Mining and Metallurgy: Melbourne).

JORC. Australasian code for reporting of identified mineral resources and ore reserves. Report of the Joint Committee of the Australasian Institute of Mining and Metallurgy, Australian Institute of Geoscientists and Minerals Council of Australia (JORC), 1999. 16 p.

NOTHOLT, A. J. G., SHELDON, R. P., DAVIDSON, D. F. Phosphate deposits of the world: phosphate rock resources. Cambridge University Press, 2005. ISBN 052167333X, 9780521673334. 596 p.

SOARES, A. O. Geoestatística para as ciências da Terra e do ambiente. Coleção Ensino da Ciência e da Tecnologia, IST Press, Lisboa, 2000, 206 p.

VELHO, J. L. Mineralogia industrial, princípios e aplicações. Lidel-edições técnicas Ltda, 2005, 606 p.

Artigo recebido em 10/03/2009 e aprovado em 17/07/2009.

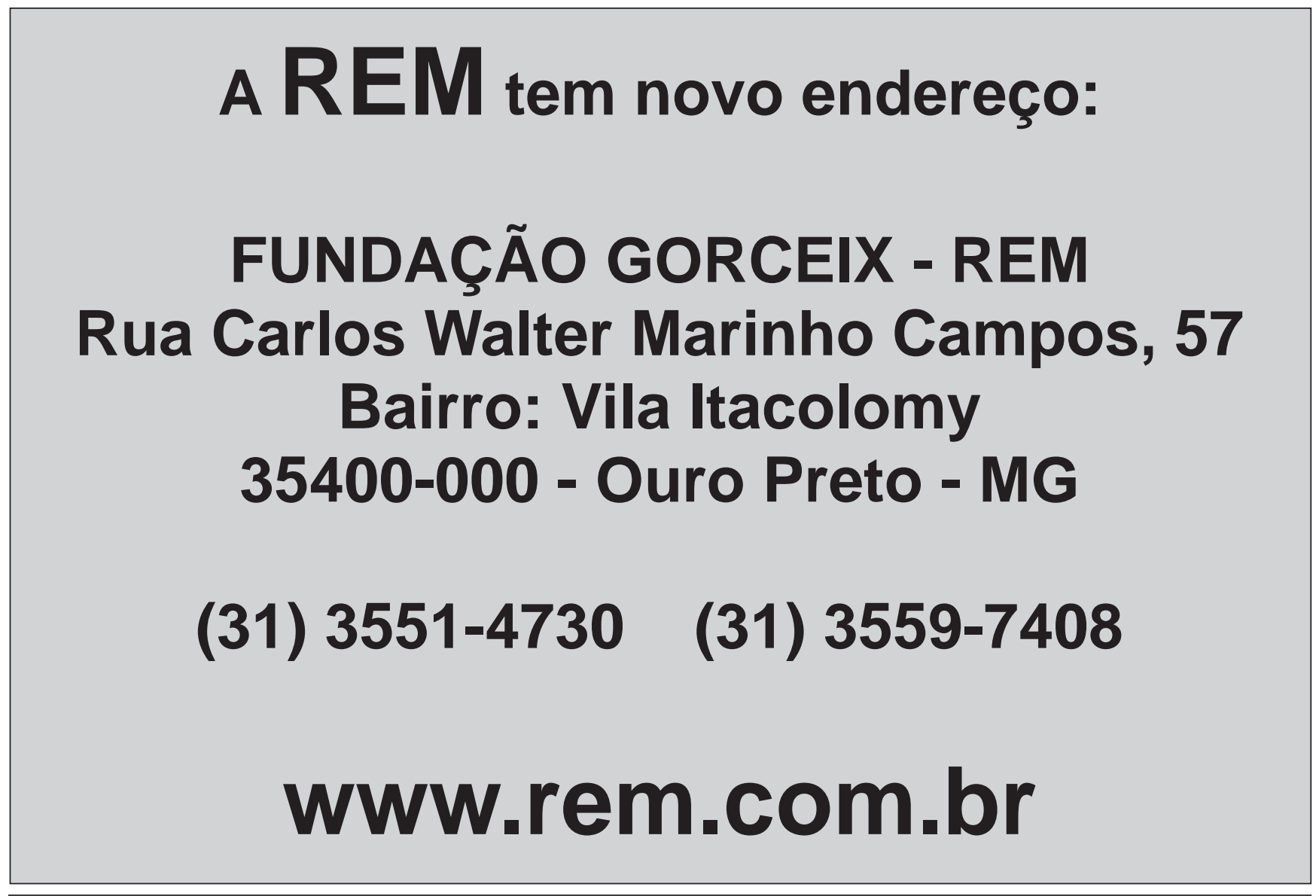

